Everyone who looks through a textbook on molecular biology is stunned by the architecture of the living cell. To a large extent this is based on the formation of flexible membranes composed of self-organizing molecules

Reinhard Lipowsky, Germany

\title{
Self-Organization of Membranes
}

1 mphiphilic molecules have two parts, A a water-soluble, hydrophilic part and a water-insoluble, hydrophobic part. Because of this, they self-assemble into layers when put in water as shown in figure 1. This "bilayer" structure represents the universal building block of all membranes found in biological systems.

Bilayer membranes are characterized by a wide range of length scales. They can be very thin with a thickness of only 4 to 5 nanometres, which is just twice the length of the amphiphilic molecules. The lateral extension of these membranes is, however, much larger and can be tens of micrometers. In order to avoid hydrophobic edges, large membrane segments form closed bags or "vesicles" which can be directly observed under the microscope.

Since membranes cover many length scales they also exhibit many levels of selforganization. On the micrometer scale they can be described as smooth surfaces, and their self-organization can be understood in terms of a few parameters such as the enclosed volume, the preferred or 'spontaneous' curvature and the bending rigidity. In general, these parameters depend on the colloidal self-organization on smaller scales. In this way, flexible membranes provide a direct connection between the nano- and the microworld.

The water volume enclosed by a vesicle is controlled by the osmotic conditions, $i e$ by the concentration of ions, molecules, and nanoparticles which are dispersed in the aqueous solution and which cannot permeate the membrane. In the dilute limit, these osmotic effects are independent of the particle size. It was realized only recently that such particles also contribute to the 'spontaneous' curvature of the membrane. This contribution depends both on the particle size and on the repulsive or attractive character of the membrane/particle interactions. Note that the particle size can be varied over several orders of magnitude from the subnano- to the microscale.

Another local mechanism which changes the "spontaneous" curvature is provided by molecules which are attached to the membranes via hydrophobic anchors. Examples are watersoluble polymer chains (such as polyethylene-glycol) with one end covalently bound to a lipid molecule. The membrane curvature arising from the anchored polymers now depends on the size of the polymers and on the membrane/polymer interactions.

Thus, using dispersed particles and/or anchored molecules, one can control both the volume of the vesicle and the 'spontaneous' curvature of its membrane in a

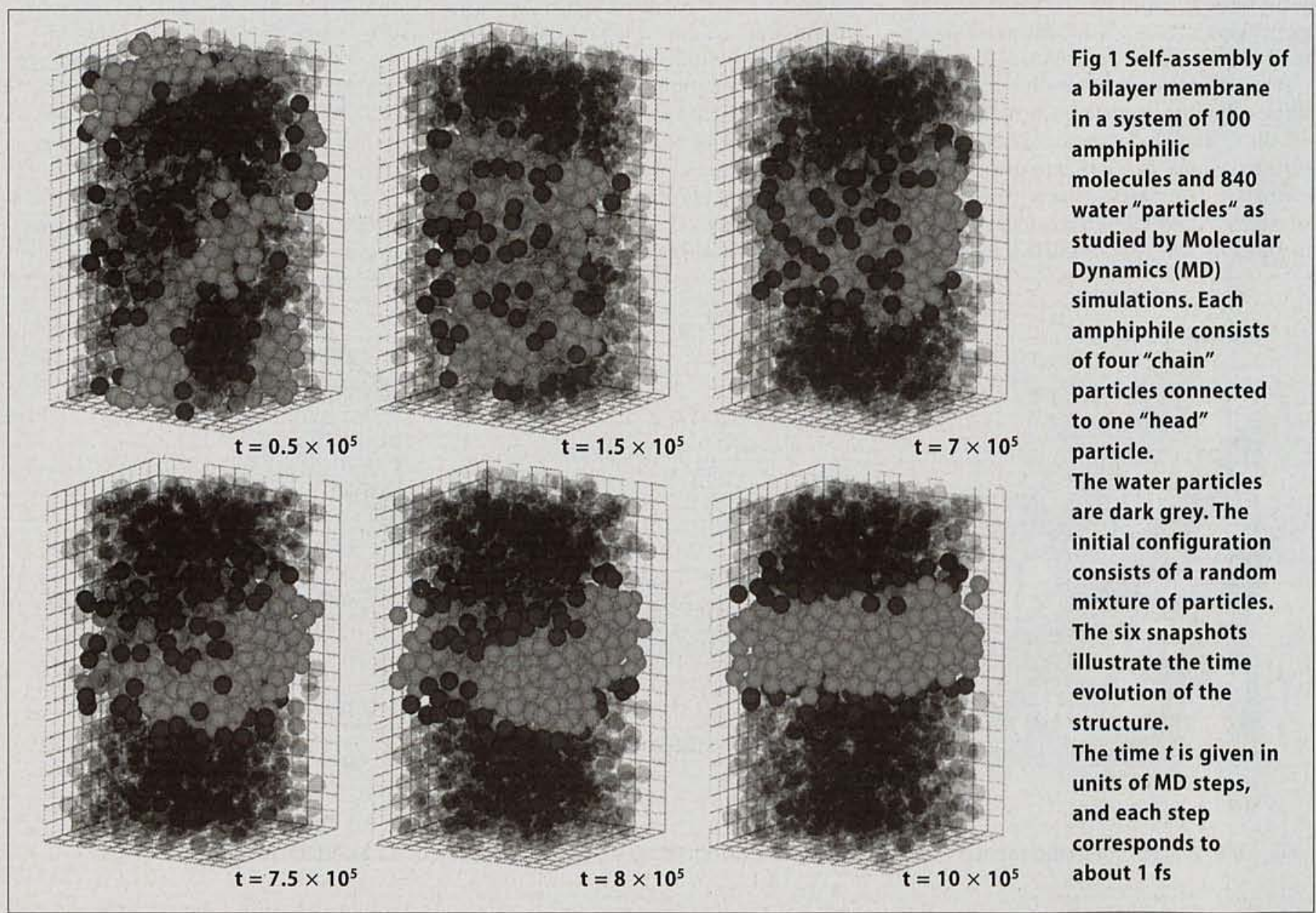


systematic way. These control parameters could be used, for instance, in order to change the vesicle shape in a cyclic fashion. Such a cycle in shape space would represent a colloidal machine which performs mechanical work on the micrometer scale. A simple example is provided by a "swimming" cycle which should lead to self propulsion at low Reynolds numbers.

Flexible membranes also undergo thermally-excited shape fluctuations in order to increase their configurational entropy. The spectrum of these fluctuations covers many wavelengths between the nanometer and the micrometer scale. On the latter scale, these fluctuations correspond to smooth changes in the membrane curvature and are governed by the bending rigidity. On the nanometer scale, the membrane should exhibit thermally-excited protrusions, ie relative displacements of individual molecules which increase the roughness of the membrane/ water interfaces.

We have recently shown that both types of fluctuations can be simultaneously studied in computer simulations using membrane models with molecular resolution. Our data provide strong evidence that the concept of bending elasticity is already meaningful on length scales which are only somewhat larger than the bilayer thickness. It was even possible to estimate the bending rigidity from the fluctuation spectrum. Somewhat surprisingly, the numerical value of the bending rigidity turns out to be consistent with a relatively simple relation in which the bending rigidity is expressed in terms of the lateral compressibility modulus and the bilayer thickness. It is highly desirable to study this relation for more complex membranes with several species of amphiphiles.

All phenomena discussed here are accessible to experiment. Thus, the self-organization of membranes provides many opportunities for fruitful interaction between theory and experiment.

The author is working at the Max Planck Institute for Colloids and Interfaces in Golm

\section{Further reading}

Molecular Biology of the Cell by B. Alberts et al, 3rd edition (Garland, New York, 1994) • Molecular Cell Biology by J. Darnell, H. Lodish, and D. Baltimore (Scientific American Books, Freeman and Company, New York, 1990) - Vesicles and Biomembranes by R. Lipowsky Encyclopedia of Applied Physics 23 pages 199 to 222 (VCH Publishers, Weinheim and New York, 1998) - R. Lipowsky, H.G. Döbereiner Europhysics Letters 43 219 (1998) - R. Goetz, G. Gompper, R. Lipowsky Physical Review Letters 82221 (1999)

\section{The study of viscous fingering - the tentacle-like flow of one fluid in another-began at the bottom of an oil well}

\author{
Anke Lindner, Daniel Bonn, Martine Ben Amar, Jacques Meunier
}

and Hamid Kellay, France

\section{Controlling Viscous Fingering}

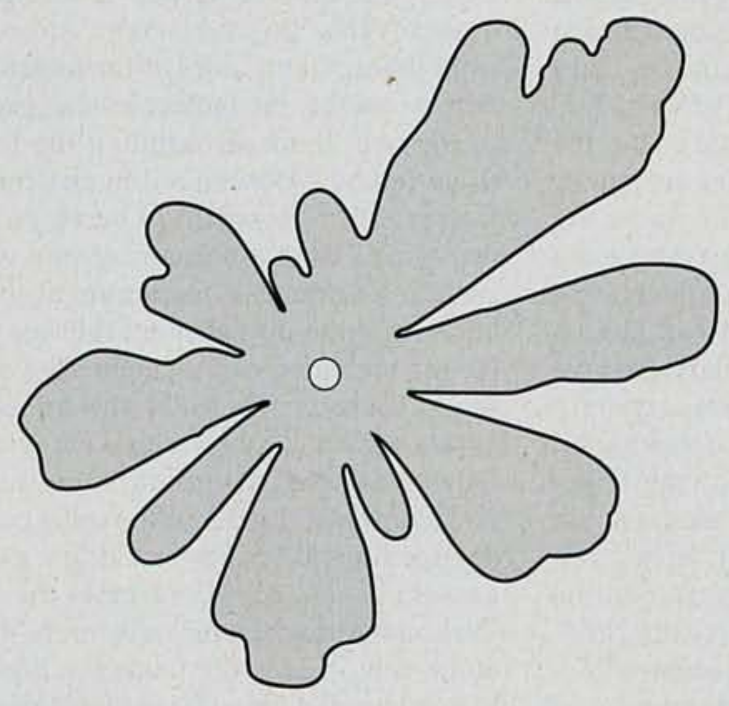

$T_{\mathrm{c}}^{\mathrm{v}}$ wo-phase flow in porous media occurs in a large number of practical situations, arguably the most important of which is oil recovery. Petroleum is generally found in porous rocks; the crude oil can be extracted from a well because it is pushed out by the high pressure in the reservoir. However, in the process of the recovery the pressure decreases rapidly and the flow of oil stops. Injection of another fluid-water or carbon dioxide gas injected below or above the oil layer respectively-allows extracting more oil from the well. As far back as 1740 in Sweden "running water was used to produce crude oil from galleries cut into the rocks bearing strata of tar and sand". In the USA this so-called "water flooding" was only recently (around 1940) recognized to be "the most efficient method". Before the second world war, it was believed that water could drown the reservoir and in many states injection of water was prohibited by law.

In such water-flooding operations, however, an hydrodynamic instability can develop at the interface between the water and the crude oil. This instability arises generically when a less viscous fluid (water or gas) pushes a more viscous one (oil) in a porous medium. As a result of the instability "fingers" of the less viscous fluid grow in the more viscous one. These fingers become narrower as the flow through the reservoir increases. This instability limits the output of a well, because if the flux becomes too high, the fingers may reach the entrance of the well rapidly, and mainly water or gas instead of oil will be recovered.

In 1956 Sir Geoffrey Taylor visited a small oil company called the "Humble Oil Company" and became interested in this problem. In a seminal paper, written jointly with P.G. Saffman, they considered an idealised version of the problem. As experiments in porous media are not easy, since they are not transparent, Taylor studied the interfacial instability of a two-phase flow in a Hele-Shaw cell: two parallel glass plates separated by a small gap $b$. This set-up models the flow in a porous medium: the same equation (Darcy's law) determines the relation between the velocity and pressure gradient in both porous media and in the Hele-Shaw cell. The average velocity of the fluid is proportional to the gradient of the pressure over the viscosity $\eta$. The coefficient of proportionality is (minus) the permeability for the porous medium and $-b^{2} / 12$ in the Hele-Shaw cell.

Indeed, in the Hele-Shaw cell the formation of "fingers" of the less viscous fluid into the more viscous fluid was ob- 